\title{
On A Harmonious Colouring Graphs And Its Applications
}

\author{
Ms.V.Kavitha ${ }^{1}$, Dr.R.Govindarajan ${ }^{2}$ \\ I Research scholar, Dravidian University, Kuppam, Asst.prof (S.G),Saveetha Engineering College, Saveetha \\ Nagar,Thandalam, Chennai-602105,India \\ 2. Associate Professor \& Head, PG \& Research Department of Mathematics, D.G. Vaishnav College \\ (Autonomous), Chennai-600106,India
}

Abstract: Harmonious colouring is a proper vertex colouring such that no two edges share the same colour pair. The harmonious Chromatic number $\chi_{h}(G)$ of a graph is the least number of colours in such a colouring. In this paper we give some new properties of harmonious colouring graphs and its applications.

Keywords: Harmonious Colouring, Harmonious chromatic Number ,Upper bound, Lower bound.

\section{Graph Colouring}

\section{Introduction}

It is more than 200 years old. It has a many practical applications like computer science, telecommunications, operation research, designs of experiments etc. It is a special case of graph labeling.

\section{Harmonious colouring}

It is a recent growing topic in the last three decades. Harmonious colouring number is used in the different families of graph such as trees, cycles, complete bipartite graphs etc. In this topic more than fifty papers are published. A harmonious Colouring of a simple graph $\mathrm{G}$ is a proper vertex colouring such that each pair of colours appears together on at most one edge. The harmonious Chromatic number $\chi_{h}(G)$ of a graph is the least number of colours in such a colouring, where $\mathrm{G}$ is a finite un directed graph with no loops and multiple edges.

Some properties of harmonious colouring are given by Frank Harary, Plant holt \& Lee [4]\& Hopcroft \& Krishnamoorthy[7] determine the harmonious Chromatic number of a graph is NP-hard and a short proof of the same result due to D.S Johnson appears in the same paper. In 1987, Sin-min Lee \& John Mitchem[16] gave its Lower bound.

In 1989, Donald G.Beare[3] he gives information about the growth rate of the harmonious Chromatic number of a family of regular graphs as a function of the number of vertices when the valency (or) the diameter is fixed and families of graphs where the harmonious coloring is minimal. In 1999, Keith Edwards [12] he define the relation between harmonious chromatic number and least positive integer.

In 1991, Zhikang Lu[19] he improved Mitchem's results on the harmonious Chromatic number of a complete binary tree and discuss the same problem for a complete trinary tree .In 1994, Keith Edwards [ 12] he improved upper bound on the harmonious Chromatic number of an arbitrary graph.

In 2006, K. Thilagavathi and J.V.Vivin [10] gives the result of harmonious chromatic number of line graph of central graph of $c_{n}, k_{n}, k_{1, n}, k_{n, n}$ and its line graphs denoted by $L\left[C\left(c_{n}\right)\right], L\left[C\left(k_{n}\right)\right], L\left[C\left(k_{1, n}\right)\right]$, $\mathrm{L}\left[\mathrm{C}\left(\mathrm{k}_{\mathrm{n}, \mathrm{n}}\right)\right]$ respectively.

\section{Definitions}

\section{Chromatic Colouring and Chromatic Number}

Let $\mathrm{G}(\mathrm{V}, \mathrm{E})$ be a graph and $\phi: V \rightarrow \mathrm{N}$ a labelling of the vertices. $\phi$ is a proper colouring if for every $\mathrm{u}, \mathrm{v} \varepsilon \mathrm{V}$ with $(\mathrm{u}, \mathrm{v}) \varepsilon \mathrm{E}$ then $\phi(\mathrm{u}) \neq \phi(\mathrm{v})$, in other words neighbouring vertices should not be assigned the same colour. The chromatic number denoted by $\chi(\mathrm{G})$.

\section{Achromatic Colouring and Achromatic Number}

The achromatic colouring of a graph is a proper vertex colouring such that each pair of colour classes is adjacent by at least one edge. The largest possible number of colours in an achromatic colouring of a graph $\mathrm{G}$ is called the and it is denoted by $\chi_{\mathrm{a}}(\mathrm{G})$. 


\section{Pseudo Achromatic Number}

The pseudo achromatic number $\alpha(\mathrm{G})$ is the maximum $\mathrm{k}$ for which there exists a complete colouring of $\mathrm{G}$. If the colouring is required also to be proper, then such a maximum is known as the achromatic number and it will be denoted here by $\chi_{a}(\mathrm{G})$.

\section{Line Distinguishing Colouring}

Let $\mathrm{G}(\mathrm{V}, \mathrm{E})$ be a graph. A colouring $\phi: V \rightarrow \mathrm{N}$ of the vertices is a line distinguishing colouring iff for every edge (u, v) $\varepsilon$ E the edge colour ( $\phi$ (u), $\phi(v))$ is unique,(i.e). it appears at most once.

\section{Harmonious Colouring and Harmonious Chromatic Number}

A harmonious colouring of a graph $G(V, E)$ is a line-distinguishing colouring which is also proper. The harmonious chromatic number of $G$ (denoted by $\chi_{\mathrm{h}}(\mathrm{G})$ ) is the smallest number $k$ such that there exists $a$ harmonious colouring of $G$ of $k$ colors.

\section{Exact coloring}

An exact coloring of a graph $\mathrm{G}(\mathrm{V}, \mathrm{E})$ is a coloring which is harmonious and complete at the same time.

\section{Central graph}

Let $\mathrm{G}$ be a finite undirected graph with no loops and multiple edges. The central graph $\mathrm{C}(\mathrm{G})$ of a graph is obtained by subdividing each edge of $\mathrm{G}$ exactly once and joining all the non adjacent vertices of $\mathrm{G}$.

\section{8. (2,n) Barbell graph}

The $(2, n)$ - Barbell graph is the simple graph obtained by connecting two copies of a complete graph $K_{n}$ by a bridge and it is denoted by $\mathrm{B}\left(\mathrm{K}_{\mathrm{n}}, \mathrm{K}_{\mathrm{n}}\right)$. From the observation of barbell graph definition .8 [ ] we define the following definition:

\section{9. $(3, n)$ Barbell graph}

The $(3, n)$ - Barbell graph is the simple graph obtained by connecting three copies of a complete graph $\mathrm{K}_{\mathrm{n}}$ by a bridge and it is denoted by $\mathrm{B}\left(\mathrm{K}_{\mathrm{n}}, \mathrm{K}_{\mathrm{n}}, \mathrm{K}_{\mathrm{n}}\right)$.

\section{0. $(\mathbf{N}, \mathbf{n})$ Barbell graph}

In general the $(\mathrm{N}, \mathrm{n})$ - Barbell graph is the simple graph obtained by connecting $\mathrm{N}$ copies of a complete graph $\mathrm{K}_{\mathrm{n}}$ by a bridge and it is denoted by $\mathrm{B}\left(\mathrm{K}_{\mathrm{n}}, \mathrm{K}_{\mathrm{n}}, \mathrm{K}_{\mathrm{n}}, \ldots \mathrm{N}\right.$ times $\left.\mathrm{K}_{\mathrm{n}}\right)$.

TABLE. 1

\section{Properties}

\begin{tabular}{|l|l|}
\hline Name of the property & Harmonious Colouring \\
\hline Upper Bound & $\begin{array}{l}\text { There are two easy but important lower bounds for } \chi_{h}(G) \text { denote the } \\
\text { maximum vertex degree of } \mathrm{G} \text { by } \Delta \text { and the number of edges of } \mathrm{G} \text { by } \mathrm{m} . \text { First } \\
\text { any vertex and all of its neighbors must receive distinct colours and this } \\
\chi_{h}(G) \geq \Delta+1 .\end{array}$ \\
\hline Lower Bound & $\begin{array}{l}\text { An upper bound for } \chi_{h}(G) \text { informs of the maximum degree of } \mathrm{G}, \Delta(\mathrm{G}) \\
\text { and } \mathrm{n} \text { the order of } \mathrm{G} ; \chi_{h}(G) \leq(\Delta \dagger 1)(\mathrm{n} 1 / 2)\end{array}$ \\
\hline Least positive integer & Let $\mathrm{Q}(\mathrm{m})$ to be the least positive integer K such that $\left(\begin{array}{l}k \\
2\end{array}\right) \geq \mathrm{m}$ then \\
& $\chi_{h}(G) \geq \mathrm{Q}(\mathrm{m})$ for any graph $\mathrm{G}$ with $\mathrm{m}$ edge. \\
\hline NP hard & The problem of harmonious colouring is NP- hard. \\
\hline
\end{tabular}


Some of the most important results on harmonious and achromatic colouring graphs, that appeared in the literature survey .For studying these particular kinds of colouring by presenting several potential applications[17].

\section{Theorem 1:}

\section{Observation}

The pseudo achromatic number $\alpha(\mathrm{G})$ is the maximum $\mathrm{k}$ for which there exists a complete colouring of $\mathrm{G}$. If the colouring is required also to be proper, then such a maximum is known as the achromatic number and it will be denoted here by $\chi_{\mathrm{a}}(\mathrm{G})$. Clearly $\chi(\mathrm{G}) \leq \chi_{\mathrm{a}}(\mathrm{G}) \leq \alpha(\mathrm{G})$, where $\chi(\mathrm{G})$ denotes, the chromatic number of $\mathrm{G}$.

\section{Theorem 2:}

Exhibiting an explicit colouring and showing a general upper bound is follows that, if $q=2^{\beta}$, for some $\beta \in I N$, and $\quad n=q^{2}+q+1$, then $q^{3}+q \leq \alpha(n) \leq q^{3}+\frac{1}{2} q^{2}+1$. Besides those implied by Bouchet's theorem, very few exact values for $\chi_{\mathrm{a}}(\mathrm{n})$ are known.

\section{Corollary 1:}

We were able to calculate exactly $\alpha(n)$ in the following family, if $q=2^{\beta}$, for some $\beta € \mathrm{IN}$, and $\mathrm{n}=$ $q^{2}+2 q+2$, then $\alpha(n)=q(n+1)=q^{3}+2 q^{2}+3 q$.

\section{Theorem 3:}

Classes of graph that can be decomposed into bounded sized components by removing a small proportion of the vertices, then such graphs of bounded degree the harmonious chromatic number is close to the lower bound $(2 \mathrm{~m})^{1 / 2}$ where $\mathrm{m}$ is the number of edges.

\section{Theorem 4:}

Let $\mathrm{G}$ be a graph and $\mathrm{H}$ be a sub graph of $\mathrm{G}$. Then $\chi_{\mathrm{a}}(\mathrm{G}) \geq \chi_{\mathrm{a}}(\mathrm{H})$.

\section{Theorem 5:}

Let $\mathrm{G}(\mathrm{V}, \mathrm{E})$ be a disconnected graph and $\mathrm{k}$ an integer number. The problem of determining whether $\chi_{\mathrm{h}}$ (G) $\geq \mathrm{k}$ is NP-complete.

\section{Theorem 6 :}

If $\mathrm{G}(\mathrm{V}, \mathrm{E})$ is a graph with $|E|=\mathrm{m}$ and $\mathrm{Q}(\mathrm{m}), \mathrm{q}(\mathrm{m})$ are defined as above then $\chi_{\mathrm{h}}(\mathrm{G}) \geq \mathrm{Q}(\mathrm{m})$ and $\chi_{\mathrm{a}}(\mathrm{G}) \leq \mathrm{q}(\mathrm{m})$, where $\mathrm{Q}(\mathrm{m})$ be the smallest positive integer $\mathrm{k}$ such that $\mathrm{m} \leq\left(\begin{array}{l}k \\ 2\end{array}\right)$ and $\mathrm{q}(\mathrm{m})$ be the greatest integer number 1 such that $\mathrm{m} \geq\left(\begin{array}{l}l \\ 2\end{array}\right)$.

Theorem 7:

Let $\mathrm{G}(\mathrm{V}, \mathrm{E})$ be a graph and $\chi(\mathrm{G})$ be its chromatic number, then $\chi(\mathrm{G}) \leq \chi_{\mathrm{a}}(\mathrm{G})$.

\section{Theorem 8:}

For any complete graph $K_{n}, \chi_{h}\left[B\left(K_{n}, K_{n}\right)\right]=2 n-1, n \geq 2$. Here $B\left(K_{n}, K_{n}\right)$ Satisfies the following properties(i)The number of vertices in $B\left(K_{n}, K_{n}\right)$ is $2 n$. (ii) The number of edges in $B\left(K_{n}, K_{n}\right)$ is $n^{2}-n+1$. (iii) The maximum degree in $B\left(K_{n}, K_{n}\right)$ is $n$. (iv) The minimum degree in $B\left(K_{n}, K_{n}\right)$ is $n-1$.

In this paper, we improved the result for 3 copies of barbell graph by our definition (9) it has given below :

\section{Theorem 9:}

For any complete graph $\mathrm{K}_{\mathrm{n}}, \chi_{\mathrm{h}}\left[\mathrm{B}\left(\mathrm{K}_{\mathrm{n}}, \mathrm{K}_{\mathrm{n}}, \mathrm{K}_{\mathrm{n}}\right)\right]=3 \mathrm{n}-2, \mathrm{n} \geq 2$

Proof:

Step 1: Let $\mathrm{G}=\mathrm{B}\left(\mathrm{K}_{\mathrm{n}}, \mathrm{K}_{\mathrm{n}}, \mathrm{K}_{\mathrm{n}}\right)$ be the Barbell graph.

Step2: By the definition, $(3, n)$ Barbell graph is obtained by connecting three copies of complete graph $\mathrm{K}_{\mathrm{n}}$ by a bridge. Let $V=\left\{v_{1}, v_{2}, v_{3} \ldots . ., v_{n}\right\}$ be the vertex set of $K^{1}, W=\left\{w_{1}, w_{2}, w_{3} \ldots \ldots, w_{n}\right\}$ be the vertex set of $K^{2}$ and $\mathrm{X}==\left\{\mathrm{x}_{1}, \mathrm{x}_{2}, \mathrm{x}_{3} \ldots \ldots, \mathrm{x}_{\mathrm{n}}\right\}$ be the vertex set of $\mathrm{K}^{3}$.

Step3: Now the colouring assignments are as follows. Since $\mathrm{K}^{1}$ contains exactly ' $\mathrm{n}$ ' vertices $(\mathrm{n} \geq 2)$ which are mutually adjacent to each other, we should colour all the $n$ vertices of ' $\mathrm{K}^{1}$ ' by $\mathrm{n}$ different colours $\mathrm{B}_{1}{ }^{\mathrm{i}}$, where 
$\mathrm{i}=1,2 \ldots$. For colouring ' $\mathrm{K}$ ', we should use ' $\mathrm{n}$ ' different colours $\mathrm{C}_{1}{ }^{\mathrm{i}}$ apart from $\mathrm{B}_{1}{ }^{\mathrm{i}}$ and any one colour from $\mathrm{B}_{1}{ }^{\mathrm{i}}, 1 \leq \mathrm{i} \leq \mathrm{n}$.

Step4: Similarly colour ' $\mathrm{K}^{3}$ ', we should use ' $\mathrm{n}$ ' different colours apart from $\mathrm{C}_{1}{ }^{\mathrm{i}}$ and any one colour from $\mathrm{C}_{1}{ }^{\mathrm{i}}, 1 \leq$ $\mathrm{i} \leq \mathrm{n}$.

Step 5 : Thus the number of colours required $=\mathrm{n}+(\mathrm{n}-1)+(\mathrm{n}-1)=3 \mathrm{n}-2$.

Example.1

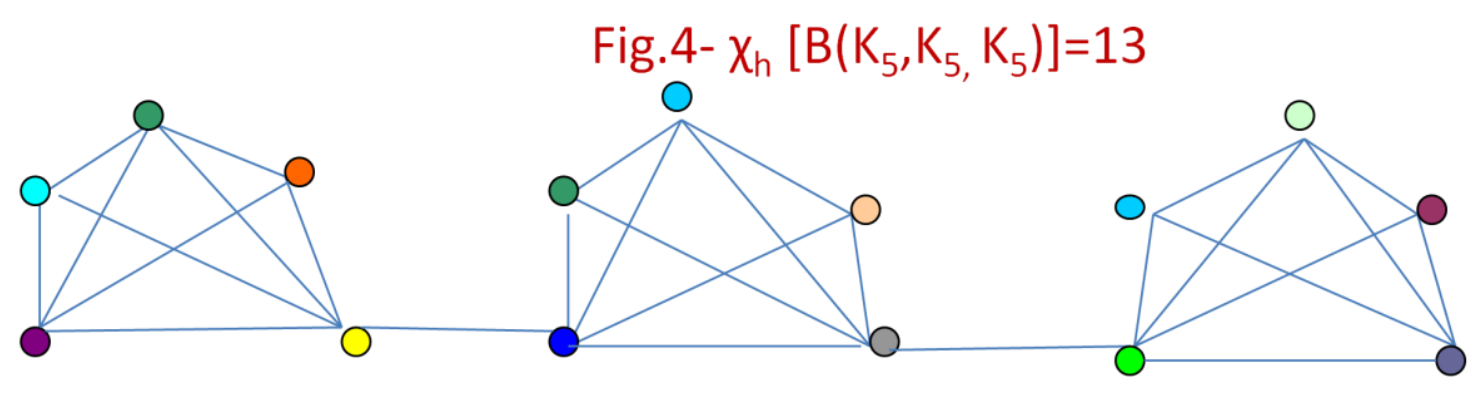

Graph theory:

V. Applications

It has many applications like, radio navigation, and image compression. In computer science, graphs are used to represent networks of communication, data organization, computational devices, the flow of computation, etc.

\section{Example.2}

The link structure of a website could be represented by a directed graph. The vertices are the web pages available at the website and a directed edge from page $A$ to page $B$ exists if and only if $A$ contains a link to $B$. A similar approach can be taken to problems in travel, biology, computer chip design, and many other fields. The development of algorithms to handle graphs is therefore of major interest in computer science.

\section{Graph colouring:}

The problem of colouring a graph has number of applications. Some of them are scheduling, bandwidth allocation ,pattern matching and puzzle Sudoku. Few examples are given as follows:

\section{List colouring:}

In the list colouring problem each vertex $\mathrm{v}$ has a list of available colours, and we have to find a colouring where the colour of each vertex is taken from its list of available colors. List colouring can be used to model situations where a job can be processed only in certain time slots, or if it can be processed only by certain machines.

\section{Multi colouring:}

A natural generalization of is to consider jobs that require more than one time slots. In the multi colouring problem each vertex $\mathrm{v}$ has a demand $\mathrm{x}(\mathrm{v})$, and we have to assign a set of $\mathrm{x}(\mathrm{v})$ colors to each vertex $\mathrm{v}$ such that neighbours receive disjoint sets of colors. Multi colouring can be used to model the scheduling of jobs with different time requirements: the set of colours assigned to vertex $\mathrm{v}$ corresponds to the $\mathrm{x}(\mathrm{v})$ time slots when we work on the job.

\section{Minimum sum colouring:}

In scheduling theory is to minimize the sum of completion times of the jobs, which is the same as minimizing the average completion time. The corresponding colouring problem is minimum sum colouring. we are looking for a colouring of the conflict graph such that the sum of the colours assigned to the vertices is minimal .Apart from trees, partial k-trees, and edges of trees, minimum sum colouring is NP-hard on most classes of graphs.

\section{Harmonious Colouring:}

It has potential applications in communication networks . For example transportation networks, computer networks. Application in radio navigation systems (ie) aviation guiding systems in bad weather 
conditions or in case of invisibility on ground objects. The airway network system is considering an optimal harmonious colouring on the graph where nodes represent the positions of the radio beacons and edges represent the airways. Also many applications like it has to be used in data compression (design of minimal hash functions) and clustering.

\section{Conclusion:}

In this paper we discussed about the Properties of harmonious chromatic number of graphs. These types of colourings presenting several applications. Also we discussed about the harmonious chromatic number of barbell graphs with $n$ copies.

\section{References}

[1] A.Chaudhary and Vishwanathan, "Approximation Algorithms for the Achromatic Number, Journal of Algorithms 41, 404-416 (2001)

[2] Colin Mc Diarmid Luo Xinhua , "Upper Bounds for Harmonious Colourings Journal of Graph Theory". Vol 15, No.6, 629-636 (1991).

[3] Donald G. Beare Norman L.Biggs Brain J.Wilson "The Growth rate of the Harmonious Chromatic Number" Journal of Graph theory Vol 13, No.3 291-299 (1989)

[4] F.Harary, S.Hedetniemi, and G.Prins, 'An interpolation theorem for graphical Homomorphism' Portugal,Math., Vol.26,pp 453$462,1967$.

[5] F.Hughes and G.MacGillivray, “ The achromatic number of graphs : a survey and some new results, Bill. Inst. Combin. Appl. 19 (1997),pp27-56.

[6] Krasikov ,Y.Roditty “ Bounds for the Harmonious chromatic Number of a Graph” Journal of graph theory Vol -18, No.2, 205-209 (1994).

[7] J. Hopcroft and M.S. Krishnomoorthy, "On the harmonious colouring of graphs", SIAM J.Algebraic Discrete methods 4(1983) 306-311.

[8] K.J.Edwards, "The harmonious chromatic number and the achromatic number of graphs", Surveys in combinatorics, London,1997,pp.13-47.

[9] K.Thilagavathi And A.Sangeetha devi, "Harmonious colouring of $\mathrm{C}[\mathrm{B}(\mathrm{Kn}, \mathrm{Kn})]$ And $\mathrm{C}[\mathrm{F} 2, \mathrm{k}]$,Proceedings of the international conference on mathematics and computer science-2009.

[10] K.Thilagavathi and J.V.Vivin, "Harmonious chromatic number of line graph of central graph. Proceedings of the international conference on mathematics and computer science-2006.

[11] K.Thilagavathi And N. Roopesh , "Achromatic colouring of line graph of central graph", Proceedings of the international conference on mathematics and computer science-2009.

[12] Kenith Edwards Colin Mc Diarmid "New upper bounds on Harmonious Colourings" Journal of graph theory Vol 18, No.3 257 -267 (1994).

[13] M.Yanakakis and F.Gavril, "Edge dominating sets in graphs", SIAM J.Appl.maths. Vol 38, pp364-372,1980.

[14] N.Cairnie and K.J. Edwards, 'Some results on the achromatic number', Journal of Graph Theory, Vol.26, pp129-136,1997.

[15] O.Frank,F.Harary and M.Plantholt, "The line- distinguishing chromatic number of a graph, Ars combin. 14(1982)241-267.

[16] Sin- Min Lee John Mitchem "An Upper bound for the Harmonious chromatic number of a graph", Journal of graph theory Vol.11 No.4 565-567 (1987).

[17] Vasiliki Mitsou, Book on “Vertex Labelings and Colorings of Graphs” The Graduate Center, The City University of New Yark.

[18] Y.Roichman, 'On the Achromatic number of Hypercubes', Journal of Combinatorial Theory,Series B,Vol.79,PP177-182, 2000.

[19] Zhikang Lu“" The Harmonious Chromatic number of a complete binary and trinary tree”, Discrete Mathematics 118 (1993) 165-172. 